\title{
Degenerative knee osteoarthritis animal model and changes in intraosseous pressure during pathological processes
}

\section{Shuo Wang}

Tianjin Hospital, Tianjin University; Chinese People's Liberation Army Joint Logistics Support Force Tianjin Rehabilitation Center (Former No. 464 Hospital of People's Liberation Army)

\section{Yu-ren Du}

Tianjin Hospital, Tianjin University

\section{Jian-xiong $\mathrm{Ma}$}

Orthpaedics Insititute of Tianjin, Tianjin Hospital

\section{Yuan Xue}

Tianjin Medical University General Hospital

\section{Wei Liu}

Tianjin Baodi Hospital

\section{Hong-chao Huang}

Tianjin Hospital, Tianjin University

\section{Lei Zhang}

Tianjin Medical University Hospital for Metabolic Diseases

\section{Xin-long Ma ( $\square$ maxinlong1962@126.com )}

Tianjin Hospital, Tianjin University https://orcid.org/0000-0001-7860-7804

\section{Research article}

Keywords: Intraosseous pressure, Articular cartilage, Subchondral bone, Degeneration, Knee osteoarthritis

Posted Date: September 3rd, 2020

DOI: https://doi.org/10.21203/rs.3.rs-32090/v2

License: (c) (i) This work is licensed under a Creative Commons Attribution 4.0 International License. Read Full License 


\section{Abstract}

Background Degenerative knee osteoarthritis (KOA) is a common clinical disease which affecting patients' quality of life. However, there is currently no standard animal model for KOA research. The purpose of this study was to find a reasonable age range of animal model for the studying of KOA pathological process, and to investigate Intraosseous pressure (IOP) in the process during different degeneration stages of KOA. Methods Male Dunkin-Hartley guinea pigs were selected and divided into groups of $3,6,9,12,18$ months old by age, 10 in each group. All knees underwent imaging examination including X-ray, Micro CT and MRI. Observed the imaging findings with the use of Kellgren-Lawrence (K-L) classification and knee osteoarthritis MRI scores. Measured the IOP of distal femur and proximal tibia in each group, and observed the differences of bilateral tibiofemoral articular cartilage in histological and immunohistochemistry, staining results were evaluated by using Mankin's score. Using one-way ANOVA and T-test were used to compare the differences indicators between groups. Results With the increase of age, changes in X-ray, Micro-CT and MRI imaging findings and pathological staining results of articular cartilage in all stages were consistent with the changing of degenerative KOA process. The IOP of distal femur and proximal tibia increased gradually with age, and reached its peak in 12-month age group, and then gradually decreased, there was a statistically significant difference of IOP between each group (femur side: $\mathrm{F}=8.261, \mathrm{P}=0.000$; tibial side: $\mathrm{F}=8.469, \mathrm{P}=0.000$ ). The IOP of the distal femur was slightly higher than that of proximal tibia, but the difference was not statistically significant $(P>0.05)$. Conclusions Dunkin-Hartley guinea pig can be used as an animal model to study different pathological stages of KOA. And there might be a correlation between the changes of IOP and the pathological progress of articular cartilage and subchondral bone in distal femur and proximal tibia.

\section{Introduction}

Knee Osteoarthritis (KOA) is a late-onset, non-specific joint-damage disease characterized by articular cartilage degeneration, subchondral bone sclerosis, bone cyst, and cartilage marginal callus formation [1]. Epidemiological investigation confirmed that the incidence of KOA is related to age, trauma, obesity, and genetic factors [2, 3], but the etiology remains unclear. The biopsy of weight-bearing articular cartilage in time series is contrary to the ethical guidelines for human trials. Therefore, it is very necessary to find suitable experimental animal models for etiological study.

The ideal KOA animal model should have the following characteristics [4]: a. Induced KOA has consistency and repeatability over the study period; b. Fully covers different stages of KOA pathological processes; c. It should be docile, economical, easy to raise and manage, and large enough to be feasible to analyze multiple factors. $d$. Therapeutic methods apply to both experimental animals and human body. However, the KOA animal model that fully meets the above criteria has not yet been discovered. Therefore, an animal model that best matches the research problem should be selected in strategy.

At present, the establishment of the KOA model is often induced by surgical means, such as knee meniscus resection or anterior cruciate ligament disconnection [2]. But osteoarthritis induced by 
traumatic procedure does not conform to the mechanism of natural degeneration. The sequence of degeneration and instability is totally opposite during the development of natural degenerative and traumatic arthritis. In a strict sense, the cause of KOA which induced by human intervention is clear and not suitable for degenerative KOA research [5].

However, the KOA of Dunkin-Hartley guinea pig naturally occurs. The degree of lesions is positively related to age and weight. This animal model does not require special modeling, it has the characteristics of docile, economical, easy to raise and management. Imaging findings shows symmetric articular cartilage lesions outside the meniscus and severe involvement of the medial tibia plateau which are very similar to those of human degenerative KOA lesions. Therefore, Dunkin-Hartley guinea pig is suitable as an experimental animal model for degenerative KOA research [6-9]. But the reasonable age range for the complete coverage of degenerative KOA pathological process is not clear. KOA in other animal models often cannot occur naturally, and usually requires surgery or other external factors to induce it, and cannot simulate and reflect the natural degeneration of human KOA.

The pathogenesis of KOA is still unclear, and controversy continues to be debated about whether it is due to bone origin, cartilage origin, synovial origin, ligament laxity or complex reasons [10]. The evidence for bone origin is that dynamic contrast-enhanced MRI found that enhanced subchondral bone signal is earlier in time than spatially corresponding cartilage lesion area, which suggesting venous outflow obstruction [11].

Intraosseous pressure (IOP) refers to the pressure generated within the marrow space. This concept is used to describe the disorder of the circulatory state in bone [12]. Intraosseous decompression has been clinically proven to effectively relieve the rest pain in KOA patients and to some extent improve knee function. There is obvious bone marrow edema in the middle and late stage of KOA, and this may be associated with local inflammatory reaction and intramedullary venous return disorder, thereby inducing an increase of IOP. Therefore, IOP might have a positive correlation with KOA pathological process, and the significance of IOP at different stages of KOA were analyzed by performing IOP measurements.

Therefore, Dunkin-Hartley guinea pigs of different ages were involved in this study. On the one hand, the standardized age range was confirmed by imaging and histology. On the other hand, the pathological process of degenerative KOA was observed by introducing the concept of IOP. The purpose is: 1 . To find a true KOA animal model; 2 . To determine the age range corresponding to the different pathological stages of degenerative KOA, in order to accurately select the suitable age of guinea pigs during KOA research; 3 . To observe the changing characteristics of IOP in the progress of KOA.

\section{Materials And Methods}

Male Dunkin-Hartley guinea pigs of 3, 6, 9, 12, and 18 months old were randomly selected, 10 for each month age. All Dunkin-Hartley guinea pigs were housed in a specific pathogen-free animal room at a temperature of $18-22{ }^{\circ} \mathrm{C}$, humidity $40-70 \%$, and 12 -hour circadian rhythm. 
The animal experiments involved in the study were approved in advance by the Experimental Animal Care and Use Committee (EACUC) of Tianjin Hospital.

1. Tibiofemoral joint (TFJ) imaging examination

\subsection{X-ray evaluation}

$10 \%$ chloral hydrate solution was intraperitoneally anesthetized, and the positive lateral radiographs of both knees were taken by UltraFocus DXA (Faxitron, US). The degree of knee degeneration was determined by Kellgren-Lawrence (K-L) classification (Table 1) [13].

\subsection{MRI evaluation}

Supine position, used the wrist joint coil to perform double-knee MRI examination (Discovery ${ }^{\mathrm{TM}} \mathrm{MRI750}$ 3.0T system, General Electric Company, USA). Since there is no MRI scoring system for small animal knee osteoarthritis, a MRI simple scoring system for guinea pig knee osteoarthritis was developed with reference to the rheumatoid MRI scoring system (RAMRIS) $[14,15]$. The system consists of three parts, including synovitis ( $0-2$ points), bone marrow edema and/or cystic changes (0-2 points), with a minimum score of 0 and a maximum score of 4 (Table 2). MRI results were determined by two experienced radiologists in a single blind method.

\subsection{Micro-CT scan}

All guinea pigs were executed and bilateral knee joints were taken for micro-CT scanning (Inveon preclinical small-animal PET/SPECT/CT system, Siemens, Germany) with $8.5 \mu \mathrm{m}$ scanning accuracy, 80 $\mathrm{kV}$ and $500 \mu \mathrm{A}$.

The anatomical region of interest (ROI) was the weight-bearing area of the patellofemoral joint. The coronal plane was marked from the anterior calcified cartilage of meniscus to the sesamoid of posteriorsuperior femoral condyle. The sagittal plane was marked between medial and lateral crest of femoral patella surface (Fig. 1).

\section{Measurement of IOP}

Checked the tightness of the pressure measuring pipeline, connected the pressure measuring tube and zeroed the dashboard. The puncture needle was used to puncture into the distal femur and proximal tibia bone marrow cavity and watched the blood return. The medial collateral ligament attachment point at both distal femur and proximal tibia were selected as the puncture point, because the position of the attachment points was relatively fixed, ensuring the accuracy and repeatability of the pressure measurement. Puncture needle ( $\varnothing 0.45 \mathrm{~mm}, \varnothing 0.7 \mathrm{~mm}, \varnothing 0.8 \mathrm{~mm}$, Hanaco Medical Co. Ltd., Tianjin, China) was punctured from the medial side direct to the opposite side of the lateral cortex (depth of penetration), the diameter of the puncture hole was $0.7 \mathrm{~mm}$. Then connected the puncture needle to the pressure measuring tube (Ø $1.8 \mathrm{~mm}$, Plastics Research Institute, Tianjin, China). Through the CSY-B ceramic thick 
film pressure sensor, the final pressure was visible on the display (Saiying Electronics Technology Development Inc., Bengbu, China). Recorded the data after the value was stable.

\section{Histological evaluation}

Observed the general specimen and evaluated the glossiness, integrity and osteophyte hyperplasia of the articular cartilage. Two experienced pathologists were simultaneously evaluated for general specimen. Articular cartilage of bilateral tibiofemoral joints (TFJ) were stained with HE, toluidine blue, Type 2 collagen and MMP13 immunohistochemical staining. The degeneration degree of articular cartilage matrix was evaluated by type 2 collagen staining, and the inflammatory response in the cartilage matrix was evaluated by the expression of MMP13. The immunohistochemical staining was performed by SABC method. Knee cartilage pathological staining scores were performed by using semi-quantitative Mankin's score [16]. But for Col-2a and MMP13, only the matrix staining section was used in the Mankin's score for scoring and statistics (Table 3) [17].

\section{Statistical analysis}

One-way ANOVA was used for analyzing the difference of knee arthritis MRI score, Mankin's score, distal femur and proximal tibia IOP changes in each group. The T-test was used for comparison between groups. The test level was taken as both sides $a=0.05$.

\section{Results}

\section{X-ray performance of bilateral TFJ}

In 3-month age group, there was no osteophyte formation of bilateral TFJ and K-L grade were all 0. In 6month age group, small osteophyte was not seen or faintly visible at medial tibial plateau, and K-L grade was 0 in 7 cases, 1 in 3 cases. In 9-month age group, small osteophyte was faintly visible at tibial plateau and intercondylar ridge, and K-L grade was 1 in 3 cases, 2 in 7 cases. In 12-month age group, the tibial plateau showed obvious osteophytes, partial TFJ space stenosis, subchondral bone sclerosis and cystic changes, and K-L grade was 2 in 2 cases, 3 in 8 cases. In 18-month age group, the tibial plateau showed obvious osteophytes, subchondral bone sclerosis and cystic changes, and the bone ends were deformed, and K-L grade were all 4 . It can be seen that with the increase of age, the K-L grade gradually increased, suggesting that osteoarthritis gradually worsened (Fig. 2).

2. MRI findings of the knee joint

In 3-month and 6-month age group, the medial posterior intercondylar fossa of femur and patellofemoral joint space were both long T1 and long T2 signals, showing synovitis-like changes, but there was no significant difference between the two groups. In 9-month age group, synovitis-like changes were located in the medial posterior side of intercondylar fossa of femur, and the signal was stronger than 3-month and 6-month age group (Fig. 3). In 12-month age group, on the basis of synovitis-like 
changes, there was a further high-signal area of the subchondral bone in the distal femur and proximal tibia, which suggesting bone marrow lesions (BMLs). In 18-month age group, synovial-like signal intensity was significantly weaker than that of 12-month age group, while BMLs in the distal femur and proximal tibia were significantly enhanced compared with 12-month age group.

The MRI scores of KOA were consistent with 3-month and 6-month age group, and the difference was not statistically significant $(P=1.000)$. Moreover, the score increased gradually after 6 months. The average score was $1.0 \pm 0.000$ point at 3 and 6 months, $1.8 \pm 0.422$ points at 9 months, $2.9 \pm 0.316$ points at 12 months, $4.0 \pm 0.000$ points at 18 -month age group. The difference was statistically significant between each group $(F=303.840, P=0.000)$.

\section{Micro-CT performance of TFJ}

In 3-month and 6-month age group, microscopic callus formation was observed in the femoral condyle and proximal tibia. No typical subchondral bone cyst formation was observed, but the density reduction zone was seen between the distal femur and the proximal tibia. In 9, 12 and 18 months age group, the thickness of the subchondral bone plate and the width of the trabecular bone increased significantly. With the increase of age, the density reduction zone between the distal femur and the proximal tibia was gradually surrounded by trabecular bone. In 18-month age group, typical subchondral bone cyst formation can be seen, which was consistent with MRI intramedullary signal characteristics (Fig. 4).

Subchondral bone cysts can be observed at both 12-month and 18-month age group, which were located at the distal femur and proximal tibia. They showed a hardened cancellous bone which was adjacent around the subchondral bone plate and was thickened and not adapted to mechanical conduction. And the typical interior has no trabecular bone structure (Fig. 4).

With the increase of age, the subchondral trabecular bone in the weight-bearing area can collapse and fuse into pieces or clumps, and can merge with the adjacent subchondral bone plate. This phenomenon was found in the distal femur and the proximal tibia weight-bearing area at 9,12 and 18 months age group (Fig. 4).

Subchondral bone cyst and subchondral trabecular collapse fusion are very similar to human KOA subchondral bone changes.

\section{Histology results of TFJ}

- General result

In 6-month age group, articular cartilage had good gloss and transparency, no obvious cartilage defects were found in the distal femoral and proximal tibia articular surface weight-bearing areas, the trochlear crest of femur and proximal tibia appeared to have cartilage-like hyperplasia; In 9-month age group, the articular cartilage had good gloss and transparency, but lower than that of 6-month age group, the trochlear crest of femur was blunt, obvious cartilage-like hyperplasia can be seen at distal femur and 
proximal tibia; In 12-month age group, articular cartilage was turbid, visible cartilage surface defects were found in the distal femur and the proximal tibia articular surface weight-bearing area, and the defects of medial condyle were particularly obvious. Visible wax-like cartilage or bone-like hyperplasia were observed at the distal femur and proximal tibia. The "U"-shaped cartilage defect in the posterior part of medial condyle of proximal tibia was formed, corresponding to the turbid color of the cartilage in the medial condyle of femur; In 18-month age group, articular cartilage was turbid, except for the distal femur and proximal tibia cartilage surface defect, partial subchondral bone was exposed at the edge of the tibial plateau. The distal femur and the proximal edge of the tibia showed obvious wax-like osteochondral hyperplasia. The "U"-shaped cartilage defect in the posterior part of medial condyle was extended to the middle and front, and the cartilage was significantly thinner (Fig. 5).

\subsection{Eosin-hematoxylin staining}

In 6-month age group, articular cartilage showed normal or mild degeneration. Normal or reduced cartilage matrix staining was limited to the cartilage surface; the distribution of chondrocytes was normal or slightly increased; the tidal line was intact (Fig. 5).

In 9-month age group, articular cartilage showed mild degeneration. The cartilage surface was slightly irregular; normal or reduced cartilage matrix staining was limited to the cartilage surface; chondrocytes can be locally enlarged, vacuolar-like degeneration, or even partial full-thickness reduction; the tidal line was intact (Fig. 6).

In 12-month age group, articular cartilage can be degenerated at various stages, with moderate degeneration being the most common. The cartilage fissure or loss extended to the middle layer or even the deep layer. The reducing of cartilage matrix staining was limited to the surface layer or extended to the middle layer, and the medial condyle was most obvious; chondrocytes can be locally increased or decreased, while chondrocytes of medial condyle were reduced; intact tidal line, intersecting with blood vessels or multiple tidal lines can be observed (Fig. 6).

In 18-month age group, articular cartilage can be degenerated at moderate-severe stages, with severe degeneration being the most common. The cartilage fissure or loss located in the middle layer or extended to the deep layer. The reducing of cartilage matrix staining extended to the middle layer or even the deep layer, and the medial condyle was most obvious; chondrocytes showed diffuse increased or focal decreased; incomplete tidal line, intersecting with blood vessels or multiple tidal lines can be observed (Fig. 6).

The Mankin's score increased with age, and the difference between the groups was statistically significant $(F=101.490, P=0.000)$. Compared with the bilateral scores in each age group, the difference was not statistically significant. ( $P>0.05$, Table 4$)$.

4.3 Toluidine blue staining 
The staining results of each age group were consistent with HE staining (Fig. 7). The Mankin's score increased with age, and the difference between the groups was statistically significant ( $F=90.405$, $P=0.000)$. Compared with the bilateral scores in each age group, the difference was not statistically significant. $(P>0.05$, Table 5$)$.

\subsection{Type 2 collagen immunohistochemical staining}

In 6-month age group, the distribution of Col-2a in articular cartilage was clear, and the focal staining of the lateral condyle cartilage weight-bearing area was reduced in the middle layer, and extended to the deep layer in medial condyle. The degree of coloration was coupled with cell proliferation, and the higher the degree of local cell proliferation, the weaker the positive staining of Col-2a (Fig. 8).

In 9-month age group, the reduction of Col-2a staining in articular cartilage was characterized by irregular distribution. The lateral condyle reduction zone was located in the surface layer and middle layer of cartilage, and reached to the deep layer in medial condyle, often accompanied by a decrease in the number of local cells. It was worth noting that the reduction of Col-2a staining may result in a jump change, that was, the deep and middle layer staining were reduced, while the surface layer was still continuous positive (Fig. 8).

In 12-month age group, the degree of reduction and involvement of the articular cartilage Col-2 staining was significantly aggravated compared with 6 and 9 months' age group. The staining reduction zone was strip or sheet shape. The lateral condyle mainly involved the surface layer, middle layer and even deep layer of cartilage, and the medial condyle obviously involved the deep layer and even the whole layer. There were also cases where the middle layer and deep layer staining reduced, while the surface layer staining enhanced (Fig. 8).

18-month age group: The degree of reduction and involvement of the articular cartilage Col-2 staining was further aggravated compared with 12-month age group.

The staining reduction zone could involve the whole layer of articular cartilage, mainly the middle and deep layers. There was no significant difference in the depth of cartilage involved of internal and external condyle (Fig. 8).

With the increasing of age, the Mankin's score gradually increased, and the difference between each age group was statistically significant $(F=25.399, P=0.000)$, and the difference between the groups of 6,9 and 12 months' age was significant (LSD-t: 6vs9, $P=0.006$; $9 v s 12, P=0.006)$, there were statistical differences between the 12 and 18 months' age group ( $P=0.028$, Table 6); there was no significant difference in the bilateral scores of the same age group $(P>0.05)$.

\subsection{MMP13 immunohistochemical staining}

In 6-month age group, the positive MMP13 mainly accumulated in distal femur and proximal tibia, the lateral edge of cartilage and the surface layer of articular cartilage. In the middle and deep layers, MMP13 
was scattered in the cells, and distributed in cells and matrix in the surface layer, which was consistent with synovitis (Fig. 9).

In 9-month age group, positive MMP13 was disseminated by the intercondylar and cartilage lateral margins to the central weight-bearing area, showing a map-like distribution, mainly involving the surface layer and intermediate layer of the articular cartilage, and can also affect the whole layer. When the middle layer and the deep layer were involved, there were positive expressions in both cells and matrix (Fig. 9).

In 12-month age group, positive MMP13 showed scattered distribution of articular cartilage, but the expression decreased, which may be related to cell degeneration, necrosis, shedding and cartilage matrix degradation. Positive MMP13 was found in cartilage exfoliation, indicating that cartilage destruction was in progress (Fig. 8).

In 18-month age group, positive MMP13 showed a full-layer distribution of articular cartilage, and MMP13 line-like deposition occurred between the middle layer, deep layer and calcified layer. This phenomenon may reflect the pathological mechanism of cartilage exfoliation (Fig. 9).

With the increase of age, the Mankin's score increased gradually. There was significant statistical difference between each age group $(F=35.120, P=0.000)$, and the difference between groups was significant between 6,9 and 12 months (LSD-t: $6 v s 9, P=0.000 ; 9 v s 12, P=0.005$ ), the difference between 12 -month and 18-month age group was statistically significant $(P=0.032$, Table 7$)$; there was no significant difference in the bilateral scores of the same age group $(P>0.05)$.

\subsection{TFJ pressure measurement}

With the increase of age, the IOP of the distal femur and the proximal tibia increased first and then decreased, which reached its highest in 12-month age group (distal femur IOP $=11.536 \pm 2.507 \mathrm{mmHg}$, proximal tibia IOP $=9.861 \pm 3.199 \mathrm{mmHg}$ ), the difference was statistically significant (femur side: $\mathrm{F}=8.261$, $P=0.000$; tibia side: $F=8.469, P=0.000$ ). Compared with 6-month age group, the IOP increased slightly in 9month age group, but the difference was not statistically significant (femur side: $P=0.997$; tibia side: $\mathrm{P}=0.973$,); Compared with 9-month age group, the IOP was significantly higher in the 12-month age group, and the difference was statistically significant (femur side: $P=0.019$; tibia side: $P=0.019$ ); However, the IOP in 18-month age group was significantly lower than that in 12-month age group, and the difference was statistically significant (femur side: $P=0.000$; tibia side: $P=0.000$ ) (Fig. 10)

In the same age group, the IOP of distal femur was slightly higher than that of proximal tibia, and the difference was not statistically significant $(P>0.05)$.

There was no significant difference in IOP between the distal femur and proximal tibia in the same age group $(P>0.05)$. 


\section{Discussion}

1. Relationship between age and the degree of KOA degeneration

Dunkin-Hartley guinea pigs can be used as degenerative KOA animal model, which has been confirmed by many studies [1-5]. However, there are still no systematic studies on the radiological and histological changes at each stage of degeneration.

In the initial stage of this study, a wide range of ages were included from the smallest of 3 months to the largest of 18 months, in order to establish a more accurate link between different ages and degeneration. In this study, the significant differences between 3-month and 6-month age group were not observed in the K-L classification, subchondral bone structure, intra-articular and intramedullary signal intensity analysis. Since the guinea pig bone matured after 6 months [18], the 3-month age group was excluded in the following experiment in order to eliminate the potential effect of bone growth on the changes of articular cartilage and subchondral bone.

According to radiographic and histological findings, the double TFJ of Dunkin-Hartley guinea pigs exhibited progressive degenerative aggravation from 6-month to 18-month age group, which with good bilateral consistency. So, it is reasonable to use this range as the classification basis for studying different degeneration stages of KOA in Dunkin-Hartley guinea pigs, and it is reliable to design the bilateral TFJ as self-controlled experiments.

The knee joint was normal or mild degeneration in 6-month age group, which was due to K-L classification, and MRI showed non-specific synovitis, although Micro-CT can detect tiny osteophytes, there was no typical subchondral bone cyst formation, and the imaging findings were consistent with histological findings and Mankin's scores. In 9-month age group, the knee was slightly degenerated due to the K-L classification, and in addition to the synovitis, MRI showed non-specific high-signal at the distal femur and proximal tibia, the signals were more diffuse. This performance echoed the density-reduced area of the corresponding part of Micro-CT, and the articular cartilage showed mild degeneration. In 12month and 18-month age group, Micro-CT showed a subchondral bone cystic area, which was more common in the medial condyle. The typical one was seen in 18-month age group. There was no bone structure remaining inside, but subchondral bone collapse fusion. MRI showed the small circular signal of the corresponding area was changed from low to high. Histological changes from 12-month to 18-month age group reflected an alternative process from reduction of surface and intermediate layer of chondrocytes, matrix decreased to deep chondrocyte proliferation. Based on the above evidence, the knee joint was moderately degenerated in 12-month age group, and was attributed to severe degeneration in 18-month age group.

The pathological changes of the tibiofemoral articular cartilage and subchondral bone of Dunkin-Hartley guinea pigs at different ages completely covered the pathological process of degenerative KOA. Selecting the appropriate guinea pig age range to study the different stages of KOA is not only conducive to the standardization of the age, but also to reduce the systemic bias caused by the different age choices. 
Subchondral bone includes subchondral cortical bone plate and cancellous bone under the bone plate. However, the subchondral bone plate is adjacent to the calcified layer of articular cartilage, and its biomechanical properties are weaker than the cortical bone of diaphysis. Below the subchondral bone plate is a porous, metabolically active cancellous bone. As KOA progresses, the calcified cartilage layer continues to thicken, the volume of articular cartilage decreases, and the subchondral bone proliferates and hardens [19-24].

The high signal zone in the subchondral bone, BMLs, refers to the edema signal located in the subchondral cancellous bone of T2 liposome phase. When the range of BMLs in the subchondral bone is limited, it is spatially identical to the subchondral bone cyst. The emergence of BMLs reflects abnormalities in subchondral bone metabolism. Histological studies confirmed that BMLs were necrotic intramedullary cells and hyperplastic fibrous connective tissue [25]. Regional subchondral trabecular bone necrosis, collapse and hyperplasia were corresponding to the degeneration of the upper articular cartilage spatially [26-27]. The intensity and extent of abnormal signal area in subchondral bone were positively correlated with the degree of articular cartilage degeneration.

Our study found that with the increase of the age, BMLs in subchondral bone marrow and subchondral bone cysts appeared in the corresponding sites of MRI and Micro-CT, and the time consistency appeared in the 12-month and 18-month age group. At that time, the articular cartilage degeneration was in moderate and severe stages. In 9-month age group, the MRI subchondral bone abnormality signal was diffuse but atypical. Micro-CT scan showed no regular shape of sclerotic bone cyst in the subchondral bone, and there was only a subchondral trabecular collapse fusion.

The collapse of the subchondral trabecular bone, the formation of bone cysts, the appearance of BMLs in the subchondral bone were all corresponded to the degree of articular cartilage degeneration. It was fully consistent with the pathological changes of subchondral bone of degenerative KOA of human. This study, on the one hand, provides powerful laboratory evidence for the use of Dunkin-Hartley guinea pigs as a degenerative KOA animal model. On the other hand, it indirectly suggests that the subchondral bone plays a crucial role in the pathological process of articular cartilage degeneration.

\section{Characteristics of IOP changes in degenerative KOA model}

$\mathrm{IOP}$ is an important physiological indicator describing the state of microcirculation within the bone. Bone tissue is a relatively closed system, and the maintenance of IOP depends on the balance between arterial perfusion and venous return. The volume change of the medullary cavity itself, like a reservoir, plays a role in regulating the basic capacity.

Our results showed that there was no significant difference in IOP between the distal femur and the proximal tibia of Dunkin-Hartley guinea pigs; the IOP of the distal femur was slightly higher than the proximal tibia, and the difference was not statistically significant. Although there is no statistical 
difference, there may be physiological significance. This difference may be related to the relatively abundant circulating blood volume in the trabecular sinus of distal femur. In addition, this difference may also have pathological significance. When the IOP of distal femur is higher than that of proximal tibia, it may cause hydrodynamic obstruction to the blood flow in the proximal trabecular bone of the tibia, the blood circulation disorder in the trabecular bone of proximal tibia, thereby a series of pathophysiological changes in the proximal tibia are further produced.

With the increase of age, the IOP of the distal femur and proximal tibia showed an upward trend first, the IOP in 9-month age group was higher than that in 6-month age group, but there was not statistically significant. It peaked at 12 months of age and fell rapidly at 18-month age group. The change of IOP firstly increased and then decreased, which was in a good agreement with the subchondral bone changes indicated by Micro-CT of each corresponding age group. The thickness and density of subchondral bone and cancellous trabeculae increased with age, and the direct result was that the mechanical elasticity of bone material and intramedullary space were both reduced. If the perfusion volume and the outflow amount are constant, the IOP will inevitably increase gradually. When the space decreases beyond the buffer range of the perfusion amount, the magnitude of increase of the IOP will increase significantly; If the subchondral bone proliferates and collapses, the volume of the medullary cavity will be reduced to a certain extent, which restrain the intra-osseous blood perfusion and further reduces the IOP. This is also the reason for the sharp decline in 18-month-old IOP in this study.

The IOP measurement site was chose at the attachment point of the medial collateral ligament to femur and tibia for the following reasons: a. The soft tissue inside the knee is relatively weak, and the anatomy is easy to be revealed; $b$. There is no important neurovascular structure in the surgical approach; $c$. There is basically no bleeding during the operation, which can avoid the inaccurate measurement of IOP caused by blood loss; $d$. The anatomical position of the medial collateral ligament of the knee is constant, which can avoid the measurement error of IOP due to non-standardization of the puncture point, and the repeatability is good.

\section{Histological characteristics of TFJ articular cartilage}

Our study found that with the growth of Dunkin-Hartley guinea pigs, chondrocytes of surface and intermediate layer of TFJ cartilage gradually decreased, however, the chondrocyte of deep layer proliferated. The acidic cartilage matrix component was progressively degraded, and the type 2 collagen fiber was reduced in the expression due to chondrocyte proliferation, and the layer disappeared. The calcified layer of cartilage was continuously thickened, and the tidal line position moved up. All of the above manifestations are the same as the classic articular cartilage degeneration pattern.

Articular cartilage fissures could be limited to the superficial and middle layer, or could be located in the deep layer alone, even in the subchondral bone plate and calcified layer cartilage, and the sinusoids invaded the deep region of the articular cartilage. This phenomenon reflected the changes in physical and chemical properties and biomechanical properties after chondrocytes and matrix components of the 
degenerative articular changed. Under the combined action of shear stress and axial pressure, the deformation ability decreased and laceration occurred in different parts.

MMP13 belongs to the family of matrix metalloproteinases (MMP), which are closely related to inflammatory responses. MMP13, also known as collagenase-3, is mainly produced by chondrocytes, synovial cells, osteoblasts and neutrophils. The ability of MMP13 is to degrade type 2 collagen in articular cartilage which is much higher than other collagenases in MMPs. More importantly, MMP13 is capable of self-catalytic activation and is central to other members of the activated MMPs family. Therefore, the small changes in MMP13 level or increased activity can amplify the decomposition of downstream protease of other MMPs [28-29].

Comparing the high expression sites of MMP13 and the degradation sites of type 2 collagen in tibiofemoral articular cartilage of Dunkin-Hartley guinea pig, which were found in good spatial consistency. In 18-month age group, MMP13 was not only expressed in the whole layer of articular cartilage, but more particularly, MMP13 appeared to deposit along the tidal line. Tidal line is the site of the non-calcified cartilage layer anchored to the calcified cartilage layer. Histologically, it is a marker separating the calcified cartilage layer and the non-calcified cartilage layer. This deposition phenomenon is very likely to clarify and predict the pathological mechanism of full layer cartilaginous exfoliation.

\section{Shortages}

At present, there is no relevant report about MRI score on knee joints of small animals such as guinea pigs. Therefore, a simple scoring system was developed for MRI quantitative assessment of KOA in our study, which was relatively simple, intuitive and high operability, but needed further verification and improvement. Besides, only the morphological description of micro-CT imaging changes was described in the ROI. Due to the large differences in organizational morphology of interest regions, not a single suitable 3D modeling method was found to accurately calculate data such as BV/TV.

In this study, the quantification such as Type 2 collagen and BMP13 were not analyzed by image software in immunohistochemical staining, which might show little significance.

\section{Conclusion}

Dunkin-Hartley guinea pigs of 6 to 18 months old could be used as reliable KOA animal model which completely covered the different pathological stages of natural degeneration of KOA through imaging and histological studies for the first time. The progress of KOA was mild at 6-month age, moderate at 912 months, and severe at 18-month age. The self-controlled study confirmed that the difference in bilateral KOA progress was small in Dunkin-Hartley guinea pigs. The characteristics of IOP changes in tibiofemoral joint were in good agreement with the pathological progress of KOA, revealing the correlation between IOP and pathological changes of articular cartilage and subchondral bone.

\section{Abbreviations}


KOA: knee osteoarthritis TFJ: tibiofemoral joints ROI: region of interest IOP: demineralized bone matrix BMLs: bone marrow lesions

\section{Declarations}

\section{Ethics approval and consent to participate}

This study was approved by the Medical Ethics Committee of Tianjin Hospital. Informed consent was obtained from all individual participants included in the study.

\section{Consent for publication}

All consents to publish from the patients who took part in this study were obtained.

\section{Availability of data and materials}

All generated or analyzed data during this study are included in this published article.

\section{Competing interests}

The authors declare that they have no competing interests.

\section{Funding}

National Natural Science Foundation of China (81572154)

\section{Authors' contributions}

SW YRD and XLM contributed to the conception and design; SW and YRD analyzed data and wrote the manuscript. XLM revised the manuscript. JXM recorded the data and revised the manuscript. YX analyzed and interpreted the data, $\mathrm{WL}$ and $\mathrm{HCH}$ assisted with the experiment. LZ collected the data. All authors read and approved the final manuscript. SW, YRD contributed equally to this work.

\section{Acknowledgements}

Many thanks are given to the colleagues of Orthopaedics Institute of Tianjin.

\section{References}

[1] Teeple E, Jay GD, Elsaid KA, Fleming BC. Animal models of osteoarthritis: challenges of model selection and analysis. AAPS J. 2013; 15(2): 438-46.

[2] Felson DT, Zhang Y. An update on the epidemiology of knee and hip osteoarthritis with a view to prevention. Arthritis Rheum. 1998; 41(8):1343-55. 
[3] Adatia A, Rainsford KD, Kean WF. Osteoarthritis of the knee and hip. Part I: aetiology and pathogenesis as a basis for pharmacotherapy. J Pharm Pharmacol. 2012; 64(5): 617-25.

[4] Little CB, Hunter DJ. Post-traumatic osteoarthritis: from mouse models to clinical trials. Nat Rev Rheumatol. 2013; 9(8): 485-97.

[5] Little CB, Smith MM. Animal Models of Osteoarthritis[J]. Current Rheumatology Reviews, 2008, 4(3): 175-182.

[6] Bendele AM. Animal models of osteoarthritis. J Musculoskelet Neuronal Interact. 2001; 1(4): 363-76.

[7] Yan JY, Tian FM, Wang WY, Cheng Y, Xu HF, Song HP, Zhang YZ, Zhang L. Age dependent changes in cartilage matrix, subchondral bone mass, and estradiol levels in blood serum, in naturally occurring osteoarthritis in Guinea pigs. Int J Mol Sci. 2014; 15(8): 13578-95.

[8] Bendele AM, Hulman JF. Effects of body weight restriction on the development and progression of spontaneous OA in guinea pigs. Arthitis Rheum. 1991; 34(9): 1180-4.

[9] Sato M, Uchida K, Nakajima H, Miyazaki T, Guerrero AR, Watanabe S, Roberts S, Baba H. Direct transplantation of mesenchymal stem cells into the knee joints of Hartley strain guinea pigs with spontaneous osteoarthritis. Arthritis Res Ther. 2012; 14(1): R31.

[10] Ameye LG, Young MF. Animal models of osteoarthritis: lessons learned while seeking the "Holy Grail". Curr Opin Rheumatol. 2006; 18(5): 537-47.

[11] Lee JH, Dyke JP, Ballon D, Ciombor DM, Rosenwasser MP, Aaron RK. Subchondral fluid dynamics in a model of osteoarthritis: use of dynamic contrast-enhancedmagnetic resonance imaging. Osteoarthritis Cartilage. 2009; 17(10): 1350-5.

[12] Arnoldi CC, Linderholm H, Müssbichler $\mathrm{H}$. Venous engorgement and intraosseous hypertension in osteoarthritis of the hip. J Bone Joint Surg Br. 1972; 54(3): 409-21.

[13] Kellgren JH, Lawrence JS. Radiological assessment of osteo-arthrosis. Ann Rheum Dis. 1957; 16(4): 494-502.

[14] Østergaard M, Edmonds J, McQueen F, Peterfy C, Lassere M, Ejbjerg B, Bird P, Emery P, Genant H, Conaghan P. An introduction to the EULAR-OMERACT rheumatoid arthritis MRI reference image atlas. Ann Rheum Dis. 2005; 64(Suppl 1): i3-7.

[15] Vasilevska V, Szeimies U, Stäbler A. Magnetic resonance imaging signs of ilitibial band friction in patients with isolated medial compartment osteoarthritis of the knee. Skeletal Radiol. 2009; 38(9): 871-5.

[16] van der Sluijs JA, Geesink RG, van der Linden AJ, Bulstra SK, Kuyer R, Drukker J. The reliability of the Mankin score for osteoarthritis. [J]. J Orthop Res. 1992; 10(1): 58-61. 
[17] Kraus VB, Huebner JL, DeGroot J, Bendele A. The OARSI histopathology initiative-recommendations for histological assessments of osteoarthritis in the guinea pig[J]. Osteoarthritis Cartilage. 2010; 18(Suppl3): S35-52.

[18] Santangelo KS, Pieczarka EM, Nuovo GJ, Weisbrode SE, Bertone AL. Temporal expression and tissue distribution of interleukin-1 $\beta$ in two strains of guinea pigs with varying propensity for spontaneous knee osteoarthritis. Osteoarthritis Cartilage. 2011; 19(4): 439-48.

[19] Milz S, Putz R. Quantitative morphology of the subchondral plate of the tibial plateau. J Anat. 1994; 185(Pt 1): 103-10.

[20] Eckstein F, Milz S, Anetzberger H, Putz R. Thickness of the subchondral mineralised tissue zone (SMZ) in normal male and female and pathological human patellae. J Anat. 1998; 192(Pt 1): 81-90.

[21] Clark JM, Huber JD. The structure of the human subchondral plate. J Bone Joint Surg Br. 1990; 72(5): 866-73.

[22] Brown TD, Vrahas MS. The apparent elastic modulus of the juxtarticular subchondral bone of the femoral head. J Orthop Res. 1984; 2(1): 32-8.

[23] Brandt KD, Myers SL, Burr D, Albrecht M. Osteoarthritic changes in canine articular cartilage, subchondral bone, and synovium fifty-four months after transection of the anterior cruciate ligament. Arthritis Rheum. 1991; 34(12): 1560-70.

[24] Dedrick DK, Goldstein SA, Brandt KD, O'Connor BL, Goulet RW, Albrecht M. A longitudinal study of subchondral plate and trabecular bone in cruciate-deficient dogs with osteoarthritis followed up for 54 months. Arthritis Rheum. 1993; 36(10): 1460-7.

[25] Zanetti M, Bruder E, Romero J, Hodler J. Bone marrow edema pattern in osteoarthritic knees: Correlation between MR imaging and histologic findings. Radiology. 2000; 215(3): 835-40.

[26] Baranyay FJ, Wang Y, Wluka AE, English DR, Giles GG, Sullivan RO, Cicuttini FM. Association of bone marrow lesions with knee structures and risk factors for bone marrow lesions in the knees of clinically healthy, community-based adults. Semin Arthritis Rheum. 2007; 37(2): 112-8.

[27] Zhai G, Stankovich J, Cicuttini F, Ding C, Jones G. Familial, structural, and environmental correlates of MRI-defined bone marrow lesions: A sibpair study. Arthritis Res Ther. 2006; 8(4): R137.

[28] Ge HX, Zou FM, Li Y, Liu AM, Tu M. JNK pathway in osteoarthritis: pathological and therapeutic aspects. J Recept Signal Transduct Res. 2017; 37(5): 431-436.

[29] Jeyakumar V, Halbwirth F, Niculescu-Morzsa E, Bauer C, Zwickl H, Kern D, Nehrer S. Chondrogenic Gene Expression Differences between Chondrocytes from Osteoarthritic and Non-OA Trauma Joints in a 3D Collagen Type I Hydrogel. Cartilage. 2017; 8(2): 191-198. 


\section{Tables}

Table 1 Kellgren-Lawrence classification

\begin{tabular}{cl}
\hline Grade & \multicolumn{1}{c}{ Radiology description } \\
\hline 0 & No radiographic features of osteoarthritis are present \\
1 & A doubtful narrowing of joint space and possible osteophytes \\
\hline 2 & $\begin{array}{l}\text { Presence of osteophytes and possible joint space narrowing on the } \\
\text { anteroposterior weight-bearing radiograph }\end{array}$ \\
\hline 3 & $\begin{array}{l}\text { Multiple osteophytes, definite joint space narrowing, sclerosis and possible bony } \\
\text { deformity }\end{array}$ \\
\hline 4 & $\begin{array}{l}\text { Large osteophytes, marked joint space narrowing, severe sclerosis, and definitely } \\
\text { bony deformity }\end{array}$
\end{tabular}

Table 2 MRI simple scoring system for KOA of Guinea pig

\begin{tabular}{ccc}
\hline Pathological manifestations & Signal characteristics & Score \\
\hline Synovitis & None & 0 \\
& High signal presence & 1 \\
\cline { 2 - 3 } Bone marrow edema and/or cystic changes & Significantly high signal & 2 \\
\cline { 2 - 3 } & None & 0 \\
\cline { 2 - 3 } & High signal presence & 1 \\
\cline { 2 - 3 } & Significantly high signal & 2 \\
\hline
\end{tabular}


Table 3 Mankin’s score system

\begin{tabular}{|c|c|c|}
\hline Parameter & Grade & Description \\
\hline \multirow[t]{7}{*}{ Articular Cartilage Structure } & 0 & Normal, smooth, uninterrupted surface \\
\hline & 1 & Mild surface irregularities \\
\hline & 2 & $\begin{array}{l}\text { fissures and/or loss of cartilage in the } \\
\text { superficial zone }\end{array}$ \\
\hline & 3 & $\begin{array}{l}\text { fissures and/or loss of cartilage extending into } \\
\text { the middle zone }\end{array}$ \\
\hline & 4 & $\begin{array}{l}\text { fissures extending into the deep zone and/or loss of } \\
\text { cartilage to deep zon }\end{array}$ \\
\hline & 5 & $\begin{array}{l}\text { Fissures or loss of cartilage extending to the } \\
\text { zone of calcified cartilage }\end{array}$ \\
\hline & 6 & Full-thickness cartilage loss \\
\hline \multirow{5}{*}{$\begin{array}{l}\text { Proteoglycan Content (staining } \\
\text { by toluidine blue) }\end{array}$} & 0 & Uniform throughout articular cartilage \\
\hline & 1 & Decreased in superficial zone only \\
\hline & 2 & Decreased in superficial and middle zones \\
\hline & 3 & Decreased in all 3 zones \\
\hline & 4 & No staining \\
\hline \multirow[t]{4}{*}{ Cellularity } & 0 & Normal (1/2 cells) \\
\hline & 1 & Diffuse/slight hypercellularity \\
\hline & 2 & Regions of hypercellularity and clustering \\
\hline & 3 & Diffuse hypocellularity \\
\hline \multirow[t]{2}{*}{ Tidemark Integrity } & 0 & Intact/single tidemark \\
\hline & 1 & Crossed by vessels/duplication of tidemark \\
\hline
\end{tabular}

Table 4 Mankin's score of Eosin-hematoxylin staining 


\begin{tabular}{cccc}
\hline Months age & Bi-total knee scores & Left knee scores & Right knee scores \\
\cline { 2 - 4 } & Mean \pm SD & Mean \pm SD & Mean \pm SD \\
\hline 6 & $2.400 \pm 3.438$ & $2.800 \pm 4.087$ & $2.000 \pm 3.082$ \\
9 & $12.800 \pm 5.095$ & $13.600 \pm 5.550$ & $12.000 \pm 5.099$ \\
12 & $29.600 \pm 8.072$ & $30.400 \pm 9.290$ & $28.800 \pm 7.662$ \\
\hline 18 & $43.200 \pm 5.029$ & $44.200 \pm 5.020$ & $42.200 \pm 5.404$ \\
\hline
\end{tabular}

Table 5 Mankin's score of Toluidine blue staining

\begin{tabular}{|c|c|c|c|}
\hline Months age & Bi-total knee scores & Left knee scores & Right knee scores \\
\hline & Mean \pm SD & Mean \pm SD & Mean \pm SD \\
\hline 6 & $3.200 \pm 4.467$ & $3.800 \pm 5.495$ & $2.600 \pm 3.715$ \\
\hline 9 & $12.800 \pm 5.029$ & $13.400 \pm 5.899$ & $12.200 \pm 4.604$ \\
\hline 12 & $27.200 \pm 7.569$ & $27.800 \pm 8.012$ & $26.600 \pm 7.987$ \\
\hline 18 & $41.200 \pm 4.467$ & $41.800 \pm 4.817$ & $40.600 \pm 4.561$ \\
\hline
\end{tabular}

Table 6 Mankin's score of Type 2 collagen immunohistochemical staining

\begin{tabular}{cccc}
\hline Months age & Bi-total knee scores & Left knee scores & Right knee scores \\
\cline { 2 - 4 } & Mean \pm SD & Mean \pm SD & Mean \pm SD \\
\hline 6 & $2.800 \pm 2.251$ & $3.200 \pm 2.387$ & $2.400 \pm 2.302$ \\
9 & $6.400 \pm 2.875$ & $6.800 \pm 3.421$ & $6.000 \pm 2.550$ \\
12 & $10.000 \pm 2.404$ & $10.400 \pm 2.510$ & $9.600 \pm 2.510$ \\
18 & $12.800 \pm 3.259$ & $13.400 \pm 3.782$ & $12.200 \pm 2.950$ \\
\hline
\end{tabular}

Table 7 Mankin's score of T MMP13 immunohistochemical staining

\begin{tabular}{cccc}
\hline Months age & Bi-total knee scores & Left knee scores & Right knee scores \\
\cline { 2 - 4 } & Mean \pm SD & Mean \pm SD & Mean \pm SD \\
\hline 6 & $4.800 \pm 1.814$ & $5.200 \pm 2.168$ & $4.400 \pm 1.517$ \\
9 & $9.600 \pm 2.319$ & $10.200 \pm 1.789$ & $9.000 \pm 2.828$ \\
12 & $12.800 \pm 3.225$ & $13.200 \pm 3.564$ & $12.400 \pm 3.209$ \\
\hline 18 & $15.200 \pm 1.989$ & $15.800 \pm 2.280$ & $14.600 \pm 1.673$ \\
\hline
\end{tabular}


Figures
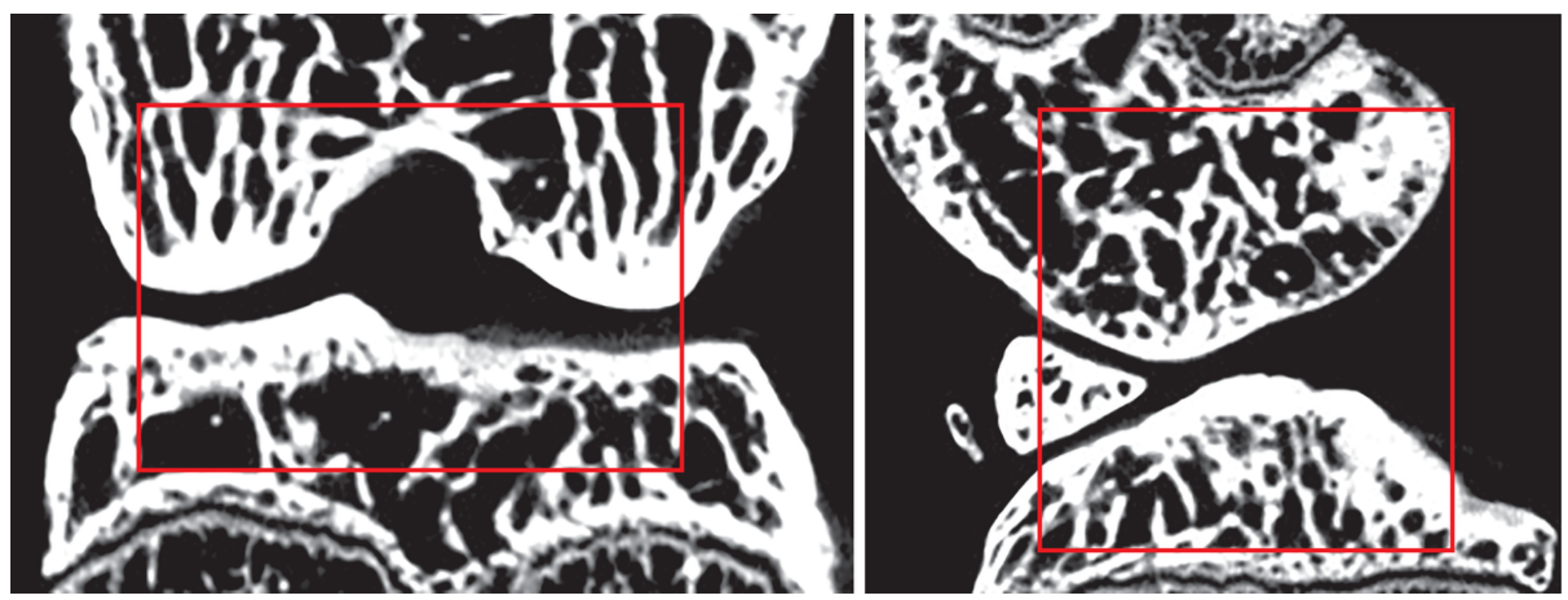

Figure 1

Anatomical regions of interest showed in red box on both coronal and sagittal planes.

3-month 6-month

9-month

12-month

18-month

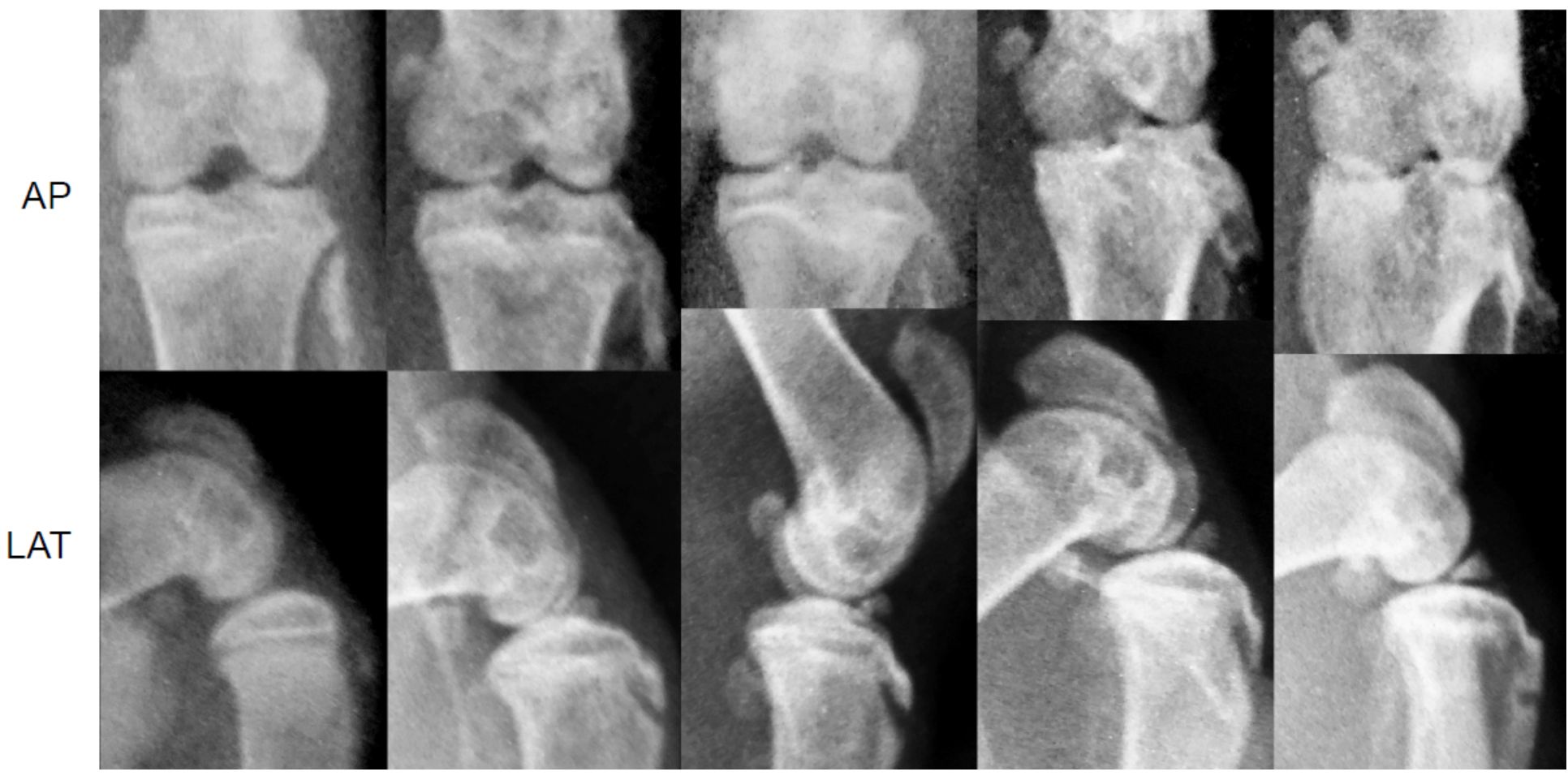

Figure 2

Anatomical regions of interest showed in red box on both coronal and sagittal planes. 


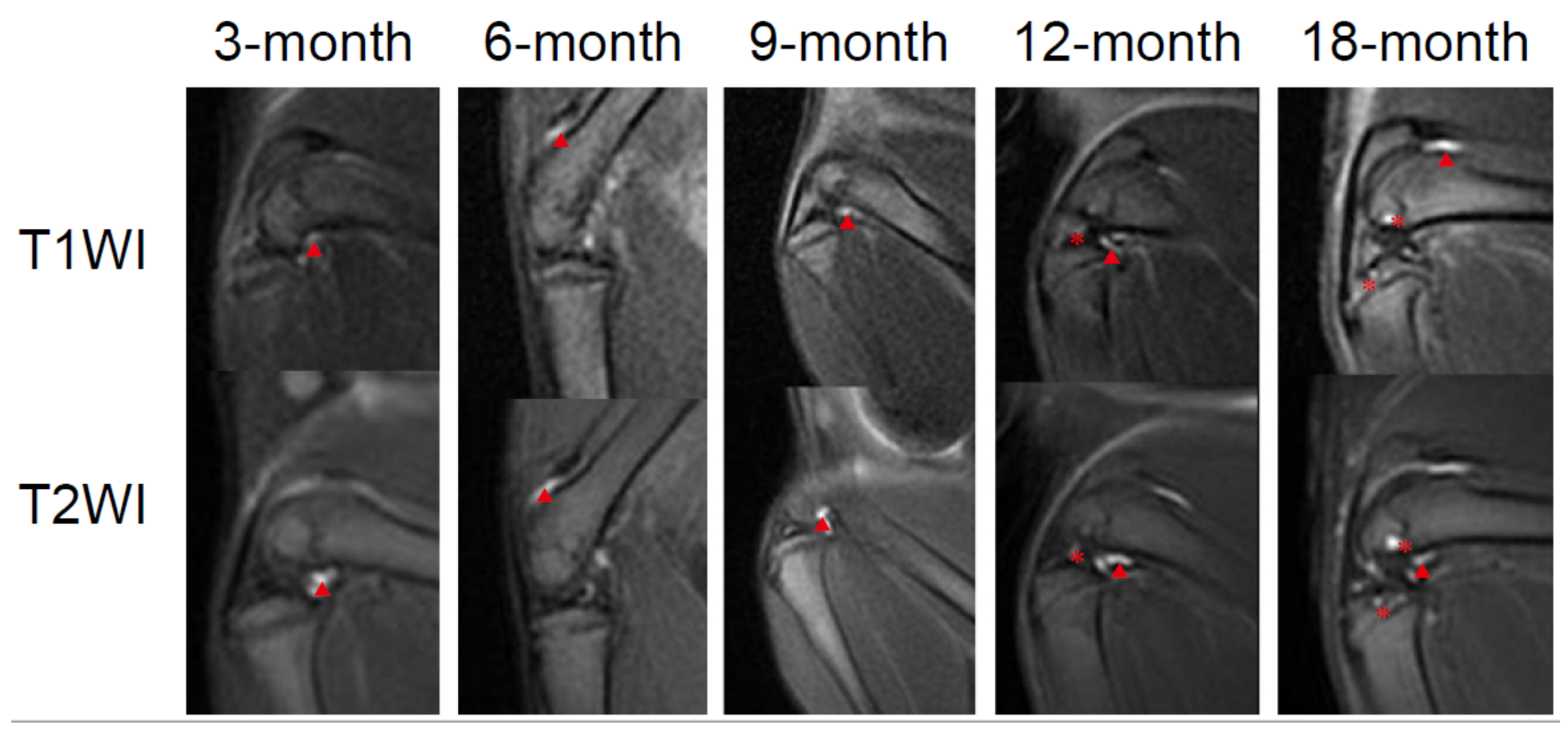

Figure 3

MRI images of right knee joints in all age groups. In 3-month and 6-month age group, the medial posterior intercondylar fossa of femur and patellofemoral joint space were both long T1 and long T2 signals, but no significant difference between the two groups. In 9-month and 12-month group, MRI showed long T1 and long T2 signal on the medial posterior side of intercondylar fossa of femur, and the signal intensity was enhanced, and Long T1 and long T2 signals appeared in the distal femur and the proximal tibia in 12-month age group. In 18-month age group, the long T1 and long T2 signal intensity in the medial posterior side of intercondylar fossa of femur was significantly weaker than that in 12-month age group, but significantly increased in the distal femur and proximal tibia. Triangle indicated synovitis-like changes, * indicated BMLs of subchondral bone.

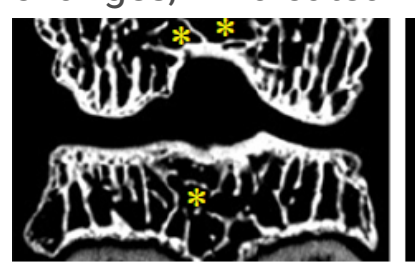

3-month

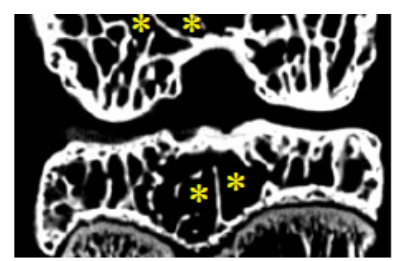

6-month

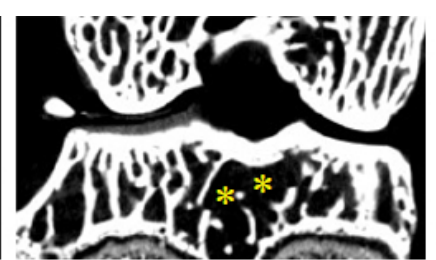

9-month

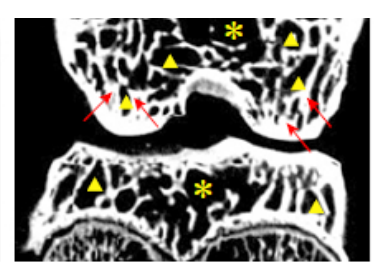

12-month

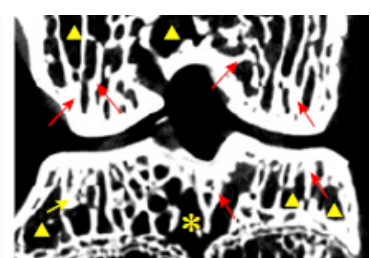

18-month

Figure 4

Micro-CT findings of right knee joints in each age group. With the increase of age, subchondral trabecular collapse fusion and subchondral bone cyst can be observed in both 12-month and 18-month age group. Triangle indicated subchondral bone cysts, * indicated density reduction zone, arrow indicated hardened cancellous bone. 


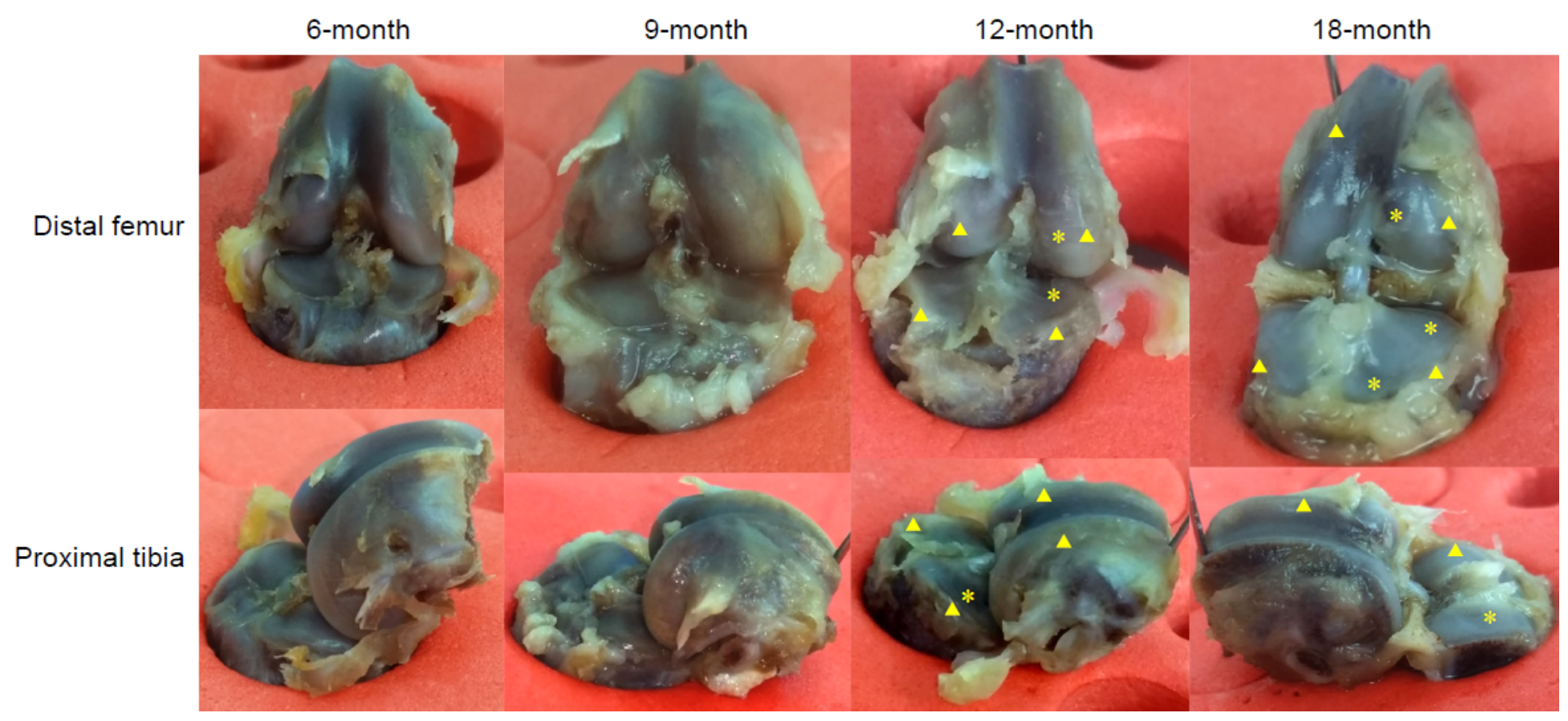

\section{Figure 5}

Micro-CT findings of right knee joints in each age group. With the increase of age, subchondral trabecular collapse fusion and subchondral bone cyst can be observed in both 12-month and 18-month age group. Triangle indicated subchondral bone cysts, * indicated density reduction zone, arrow indicated hardened cancellous bone.

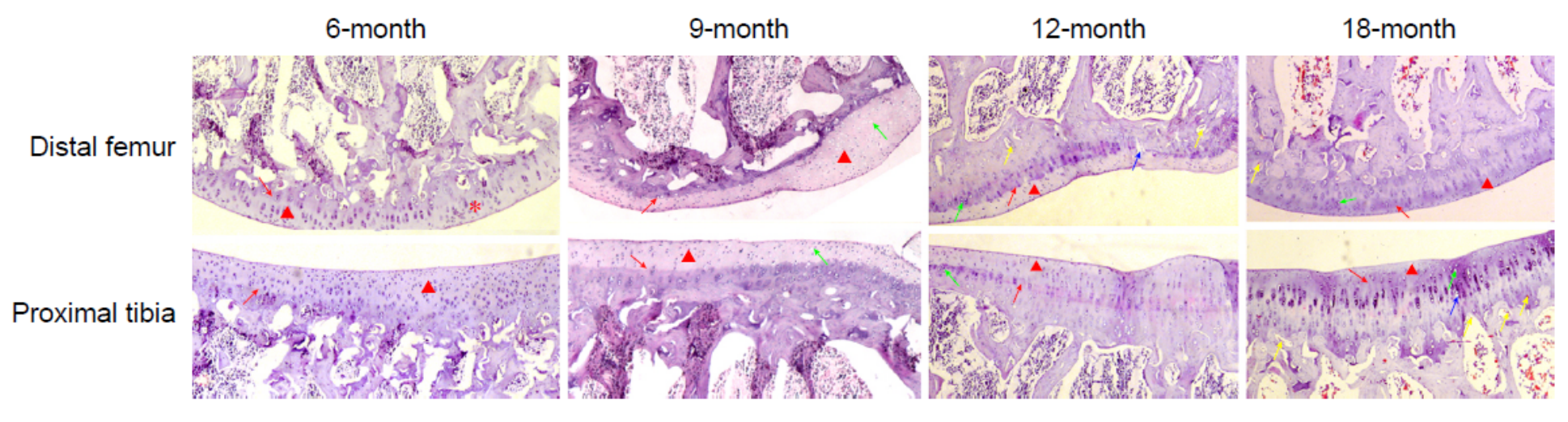

\section{Figure 6}

HE staining of right knee joint in each age group. With the increase of age, cartilage fissures or loss, chondrocyte reduction and hyperplastic changes, cartilage matrix coloration level change can be observed. Triangle indicated cartilage matrix, red arrow indicated tidal line, green arrow indicated vacuolar-like degeneration, blue arrow indicated cartilage fissure, yellow arrow indicated blood vessels. ( $\times$ 40) 


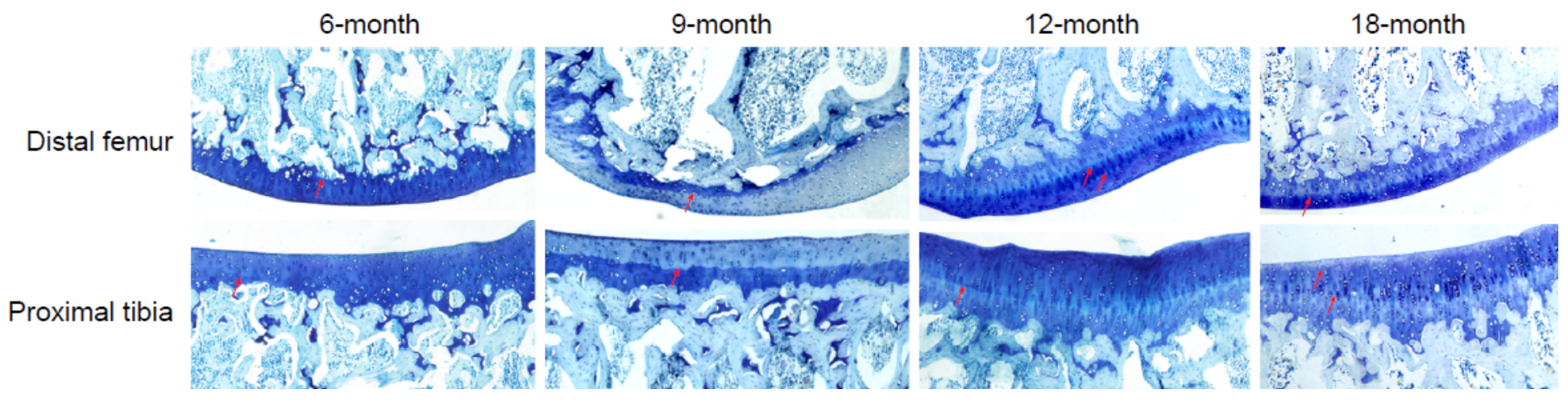

\section{Figure 7}

Toluidine blue staining of right knee joint in each age group. Red arrow indicated tidal line. $(\times 40)$

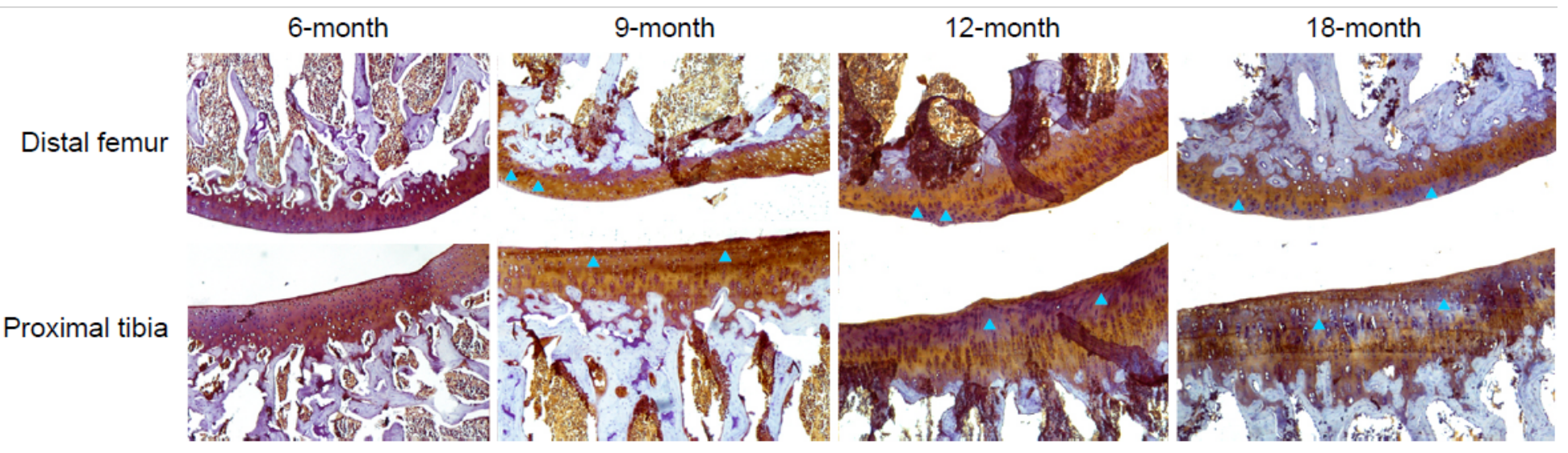

\section{Figure 8}

Col-2a staining of right tibiofemoral joint cartilage in each age group. With the increase of age, the reduction range and destruction level of Col-2a staining gradually increased. Blue triangle indicated staining reduction zone. $(\times 40)$

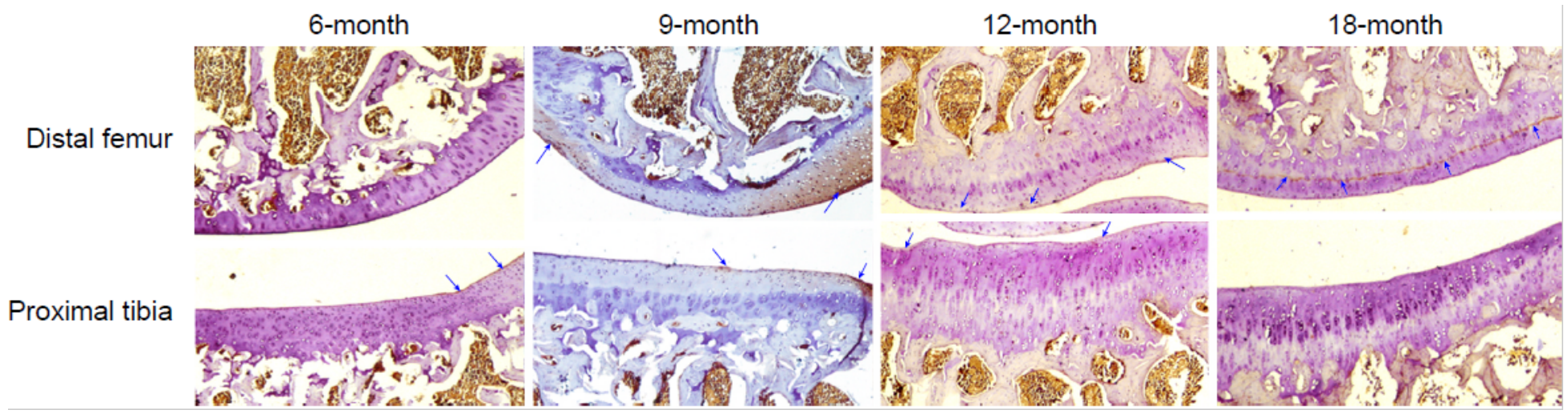

\section{Figure 9}

MMP13 staining of right tibiofemoral joint cartilage in each age group. The main distribution level and expression density of MMP13 in cartilage were positively correlated with age. Arrow indicated MMP13 positive expression region. $(\times 40)$ 

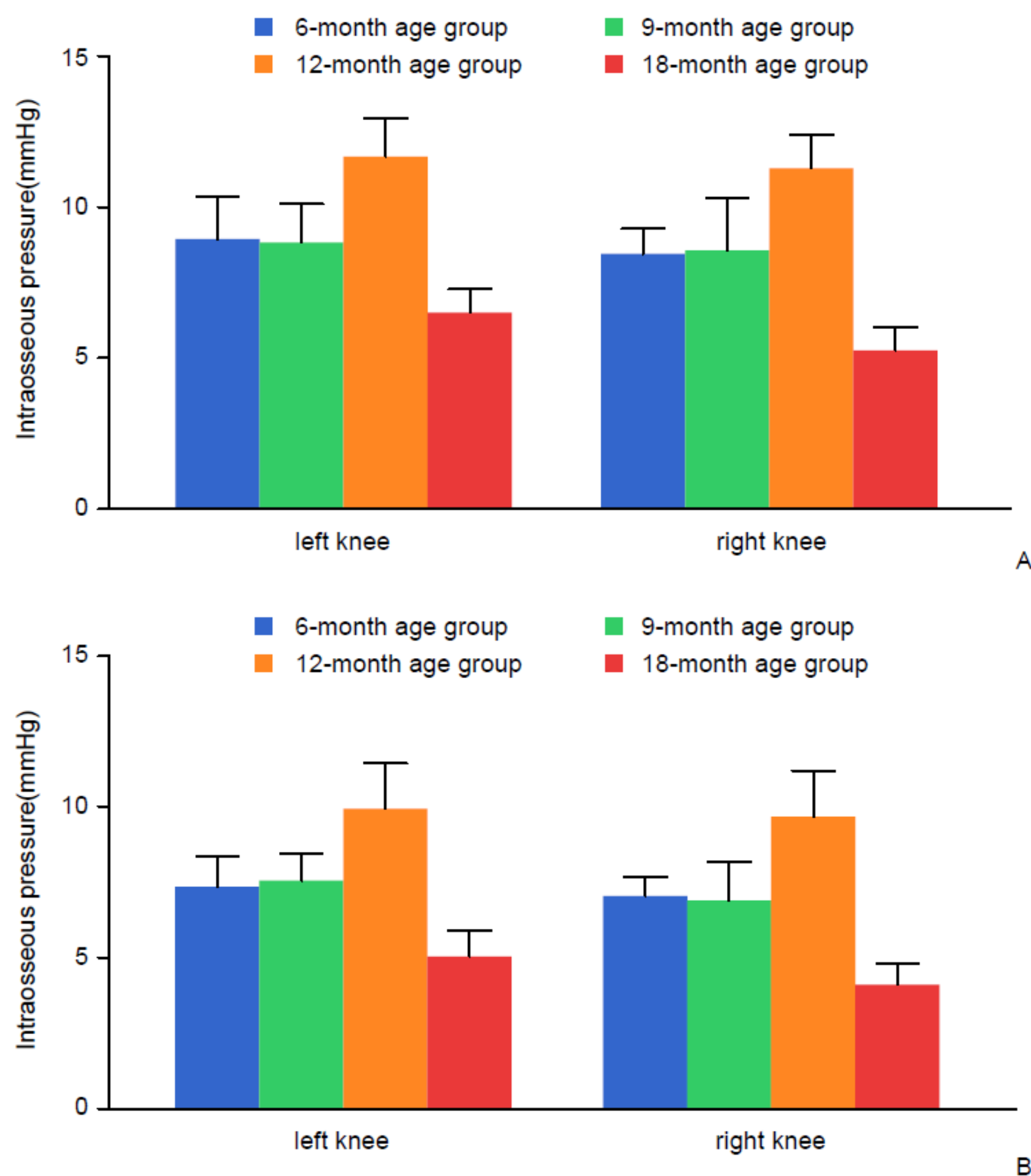

Figure 10

IOP of bilateral tibiofemoral joint in each age group. A. IOP of distal femur in each age group. B. IOP of proximal tibia in each age group. With the increase of age, the IOP of the distal femur and proximal tibia were consistent, and the IOP gradually rose at the beginning. The IOP of the 9-month age group to $12-$ month age group showed a rapid increasing trend. However, in 18-month age group, the IOP rapidly dropped below the normal level. 